\title{
PACS-1 Mediates Phosphorylation-Dependent Ciliary Trafficking of the Cyclic-Nucleotide-Gated Channel in Olfactory Sensory Neurons
}

\author{
Paul M. Jenkins, ${ }^{1}$ Lian Zhang, ${ }^{1}$ Gary Thomas, ${ }^{2}$ and Jeffrey R. Martens ${ }^{1}$ \\ ${ }^{1}$ Department of Pharmacology, University of Michigan, Ann Arbor, Michigan 48109-5632, and ${ }^{2}$ Vollum Institute, Oregon Health \& Science University, \\ Portland, Oregon 97239
}

Impaired ciliary protein transport in olfactory sensory neurons (OSNs) leads to anosmia, and is a newly recognized clinical manifestation of a class of human disorders called ciliopathies. Surprisingly little is known regarding the mechanisms controlling trafficking to this unique neuronal compartment. Here, we show a novel role for phosphofurin acidic cluster-sorting protein 1 (PACS-1) in the ciliary trafficking of the olfactory cyclic-nucleotide-gated (CNG) channel. PACS-1 is an intracellular sorting protein that mediates its effects through the binding of acidic clusters on cargo protein. This interaction is dependent on CK2 phosphorylation of both PACS-1 and its cargo. We show that CNGB1b contains two putative PACS-1 binding sites, which are phosphorylated by the serine/threonine protein kinase, CK2. Additionally, we show that PACS-1 is expressed in OSNs and interacts in complex with the CNG channel. CK2 inhibition in native OSNs causes a loss of CNG channel from cilia and subsequent olfactory dysfunction, while adenoviral expression of mutant PACS-1 causes similar mislocalization. These results provide a mechanism for the subunit-dependent ciliary trafficking of the CNG channel and offer insight into the mechanisms of ciliary transport.

\section{Introduction}

Cilia are microtubule-based organelles that can project from the surface of nearly any mammalian cell. Defects in cilia structure and function have been implicated in a growing number of human diseases involving multiple organ systems (Pazour and Rosenbaum, 2002; Badano et al., 2006; Christensen et al., 2007). Many neuronal cells possess primary cilia; however, their precise function often remains obscure (Fuchs and Schwark, 2004). The olfactory system is one neuronal system that is critically dependent on proper ciliary function. Olfactory sensory neurons (OSNs) are unique in that cilia project from multiple basal bodies in their apical dendrite to make contact with the external environment (Menco, 1994). These cilia compartmentalize signaling molecules, thereby allowing rapid and efficient activation of the odorant cascade.

Since cilia lack protein synthesis machinery, proteins must be produced in the cell and transported into the cilium via intraflagellar transport (IFT) (Rosenbaum and Witman, 2002;

\footnotetext{
Received April 1, 2009; revised June 25, 2009; accepted July 20, 2009.

This work was supported by National Institutes of Health Grants DC009606 (J.R.M.), DK37274 (G.T.), Al49793 (G.T.), GM007767 (P.M.J.), and DC00011 (P.M.J.) and National Research Service Award Fellowship DC009524 (P.M.J.). We thank Kristen Verhey, Ben Margolis, and Toby Hurd (University of Michigan) for helpful discussions, critical review of the manuscript, and reagents. We also thank Donna Martin and her laboratory (University of Michigan) for assistance with the in situ hybridization as well as Margaret Gnegy and her laboratory (University of Michigan) for assistance with the in vitro phosphorylation experiments. We would like to acknowledge Randall Reed and his laboratory (Johns Hopkins University, Baltimore, MD) for the generous gift of the ACIII-GFP plasmid.

Correspondence should be addressed to Asst. Prof. Jeffrey R. Martens, Department of Pharmacology, University of Michigan, 1150 West Medical Center Drive, 1301 MSRB III, Ann Arbor, MI 48109-5632. E-mail: martensj@umich.edu.

D0I:10.1523/JNEUROSCI.1590-09.2009

Copyright $\odot 2009$ Society for Neuroscience $\quad$ 0270-6474/09/2910541-11\$15.00/0
}

Scholey, 2003). Entry of this cargo into the cilium is regulated by a protein complex at the basal body (Rosenbaum and Witman, 2002; Scholey, 2003). Alterations in the localization of ciliary proteins have been shown to underlie multiple human diseases including olfactory dysfunction, polycystic kidney disease, retinal degeneration, and primary ciliary dyskinesia (Pazour and Rosenbaum, 2002; Kulaga et al., 2004; Otto et al., 2005; Michalakis et al., 2006; Christensen et al., 2007; McEwen et al., 2007). Despite advances in the understanding of cilia formation and function, we still know little about the mechanisms regulating entry into this compartment.

All of the necessary components of the olfactory signal transduction cascade are enriched in cilia, including odorant receptors, G-proteins, adenylyl cyclase III (ACIII), and the cyclicnucleotide-gated (CNG) channel (Ronnett and Moon, 2002). The olfactory CNG channel comprises three channel-forming subunits (CNGA2, CNGA4, and CNGB1b). Channel activation allows the initial depolarization of the OSN, and mislocalization of the CNG channel from cilia has been implicated in diseases, including anosmia and retinal degeneration (Hüttl et al., 2005; Michalakis et al., 2006). Previously, we have shown that the CNGB1b subunit is necessary for ciliary localization of the olfactory CNG channel (Jenkins et al., 2006); however, the mechanism of this subunit-dependent ciliary trafficking remained unknown.

Recently, the intracellular trafficking protein PACS-1 has been shown to localize to the base of human respiratory cilia and control the localization of nephrocystin 1 to the transition zone of respiratory cilia (Schermer et al., 2005). While PACS-1 has been shown to interact with acidic cluster-containing ion channels such as polycystin-2/TRPP2, TRPV4, and CLC-7 (Kulaga et al., 2004; Youker et al., 
2009), no direct role has been demonstrated in the control of ciliary localization of ion channels or other neuronal cargo.

Here, we found that PACS-1 interacts with CNGB1b and is required for the control of its ciliary localization. Additionally, we found that CK2 phosphorylation of both PACS- 1 and CNGB1b is necessary for this ciliary localization. These results represent the first report of a role for PACS-1 in the ciliary localization of membrane proteins, and highlight a novel mechanism for the control of neuronal cargo ciliary localization.

\section{Materials and Methods}

Antibodies. Monoclonal anti-acetylated $\alpha$ tubulin clone 6-11 B-1 (1: $1000)$ and anti-flag clone $M 2$ antibodies ( $1 \mu \mathrm{g}$ for immunoprecipitation), as well as polyclonal anti-flag (1:500) antibodies, were from SigmaAldrich. Monoclonal anti-PACS-1 clone 4 (1:500) antibodies were from BD Biosciences. Polyclonal anti-CNGA2 (1:200) antibodies were from Alomone Labs. Monoclonal anti-CNGA2 clone CRO2H7 (1:250) was a gift from Dr. U. Benjamin Kaupp (Institut für Strukturbiologie und Biophysik, Strukturbiochemie, Forschungszentrum Jülich, Jülich, Germany). Polyclonal anti-HA (1:500 for immunostaining, $1 \mu \mathrm{g}$ for immunoprecipitation) antibodies were from Santa Cruz Biotechnology. Rat anti-c-myc clone JAC6 (1:500) antibodies were from AbD Serotec. AlexaFluor (405, 594, or 647)conjugated goat anti-mouse IgG, goat anti-rabbit IgG, and goat anti-rat IgG secondary antibodies (1:1000) were from Invitrogen.

Cell culture. Madin Darby canine kidney (MDCK) cells were cultured, transfected, and prepared as described previously (Jenkins et al., 2006). Briefly, MDCK cells were grown for $7 \mathrm{~d}$ after confluence on Transwell filter supports (Corning Costar) and transfected with $0.5 \mu \mathrm{g}$ per DNA construct and Lipofectamine plus reagent (Invitrogen) according to the manufacturer's protocol. HEK293 cells were cultured in DMEM containing $10 \%$ fetal bovine serum, $100 \mathrm{U} / \mathrm{ml}$ penicillin, and $100 \mu \mathrm{g} / \mathrm{ml}$ streptomycin at $37^{\circ} \mathrm{C}$ and $5 \% \mathrm{CO}_{2}$. For immunoprecipitation, HEK293 cells were plated on $100 \mathrm{~mm}$ dishes at $50 \%$ confluence and transfected with $1 \mu \mathrm{g}$ of cDNA and $3 \mu \mathrm{l}$ of Lipofectamine 2000 (Invitrogen) according to the manufacturer's protocol. CNGB1b serine to alanine mutations were performed using the QuikChange Site-Directed Mutagenesis Kit purchased from Stratagene according to the manufacturer's protocol.

Adenovirus preparation. PACS-1 S278A IRES eGFP was cloned into the adenoviral shuttle plasmid, pDC315, and adenovirus was prepared using the Admax Adenoviral Preparation Kit (Microbix Biosystems) according to the manufacturer's protocol. Adenovirus was amplified by infection of HEK293 cells in $150 \mathrm{~cm}^{2}$ flasks, which were harvested at $\sim 50 \%$ cytopathic effect, and virus was purified using the Adeno-X Maxi Prep Kit (Clontech) according to the manufacturer's protocol. Virus was dialyzed into a solution of $2.5 \%$ glycerol, $25 \mathrm{~mm} \mathrm{NaCl}$, and $20 \mathrm{~mm}$ Tris$\mathrm{HCl}, \mathrm{pH} 8.0$, and stored at $-80^{\circ} \mathrm{C}$ until use. Viral titer was calculated using an adenoviral plaque assay according to Adeno-X Maxi Prep Kit instructions.

Intranasal injection. All animals were handled according to the guidelines for animal care at the University of Michigan. Adult CD1 mice (2-5 months) were anesthetized by intraperitoneal injection with $100 \mathrm{mg} / \mathrm{kg}$ ketamine and $5 \mathrm{mg} / \mathrm{kg}$ xylazine. Animals were placed in the supine position and PE10 tubing was inserted into the right nostril to a depth of 7 $\mathrm{mm}$. Adenoviral solutions were delivered in a total volume of $50 \mu \mathrm{l}$ of sterile $0.9 \%$ saline mixed with $0.02 \%$ fast green dye at a rate of $4 \mu \mathrm{l}$ per minute $\left(2.5 \times 10^{8}\right.$ pfu for PACS-1 S278A IRES eGFP adenovirus; $1 \times$ $10^{9} \mathrm{pfu}$ for eGFP adenovirus). Treatments were repeated for 3 consecutive days. Drug delivery was performed in a total volume of $25 \mu \mathrm{l}$ of sterile $0.9 \%$ saline mixed with $0.02 \%$ fast green dye at a rate of $4 \mu \mathrm{l}$ per minute [ $\pm 20 \mu \mathrm{M}$ CK2 inhibitor, 4,5,6,7-tetrabromotriazole (TBB)]. Animals were allowed $30 \mathrm{~min}$ to recover from anesthesia on their backs before being returned to their home cage. Mice infected with virus were allowed $4 \mathrm{~d}$ for expression of ectopic protein. Those injected with CK2 inhibitor were allowed $4 \mathrm{~h}$ of recovery.

Tissue preparation. For immunostaining, adult CD1 mice (2-5 months) were deeply anesthetized and killed by cardiac perfusion with 50 $\mathrm{ml}$ of PBS followed by $50 \mathrm{ml}$ of $4 \%$ paraformaldehyde (PF) at a rate of 10 $\mathrm{ml}$ per minute. Olfactory epithelium $(\mathrm{OE})$ was removed and postfixed in
$4 \% \mathrm{PF}$ for $1 \mathrm{~h}$ at $4^{\circ} \mathrm{C}$, cryoprotected in $30 \%$ sucrose overnight at $4^{\circ} \mathrm{C}$, and embedded in OCT compound (Tissuetek). Cryostat sections were sliced at a thickness of $20 \mu \mathrm{m}$ and allowed to dry onto Superfrost Plus slides (Fisher Scientific) for $20 \mathrm{~min}$ at room temperature.

Immunostaining. Olfactory epithelium sections were permeabilized in PBS containing $0.3 \%$ Triton X-100 and $2 \%$ goat serum for 20 min at room temperature. MDCK cells were fixed for $7 \mathrm{~min}$ with $4 \% \mathrm{PF}$, permeabilized for 10 min in PBS containing $0.1 \%$ Triton X-100, and blocked in PBS with 2\% goat serum for at least $10 \mathrm{~min}$. Samples were incubated in primary antibodies diluted in 2\% goat serum PBS for $1 \mathrm{~h}$ at room temperature and washed three times for $2 \mathrm{~min}$ in PBS (anti-CNGA2 antibody incubation was overnight at $4^{\circ} \mathrm{C}$ in humidified chamber). Samples were incubated in fluorescently conjugated secondary antibodies diluted in $2 \%$ goat serum/PBS for $1 \mathrm{~h}$, washed three times for $2 \mathrm{~min}$ in PBS, and mounted using Prolong Gold anti-fade reagent (Invitrogen).

Confocal imaging. Confocal imaging was performed as described previously (Jenkins et al., 2006). Images of transfected cells displaying fluorescent signals were acquired on an Olympus Fluoview 500 confocal microscope with a $60 \times 1.40$ numerical aperture (N.A.) or $100 \times 1.35$ N.A. oil objective. Exposures were adjusted so that the maximal pixel intensities were at least half saturation. Images were obtained by taking a series of stacks every $0.5 \mu \mathrm{m}$ through the cell (generally 3-5 $\mu \mathrm{m}$ ) and combining the images into a composite stack. For MDCK cell experiments, while the entire confocal stack containing sections through the whole cell was used to generate images for display, fluorescence intensity analysis and colocalization were measured in images generated only from focal planes containing cilia (supplemental Fig. 3, available at www. jneurosci.org as supplemental material). For imaging, we used a $405 \mathrm{~nm}$ laser diode with a $430-460 \mathrm{~nm}$ bandpass filter, a $488 \mathrm{~nm}$ laser with a $505-525 \mathrm{~nm}$ bandpass filter, a $543 \mathrm{~nm}$ laser with a $560 \mathrm{~nm}$ long-pass filter, and a $633 \mathrm{~nm}$ laser with a $660 \mathrm{~nm}$ long-pass filter. Fluorescent signals of compressed $Z$-stacks were quantified using NIH ImageJ software. For adenoviral OE experiments, to minimize contribution of ciliary protein localization from uninfected OSNs, we used compressed $Z$-stacks corresponding only to the thickness of the infected OSN. Images were analyzed with ImageJ software (NIH), and statistics were performed with Prism 5 software from Graphpad Prism Software. Adjustments of contrast and brightness were performed using Adobe Photoshop 9.0.

In situ hybridization. In situ hybridization was performed on mounted coronal sections of p1.5 CD1 mouse head using an 800 bp digoxigeninlabeled, antisense PACS-1 riboprobe or the control, sense probe, with a hybridization temperature of $65^{\circ} \mathrm{C}$ as described previously (Martin et al., 2002). Eight hundred nucleotides of cDNA corresponding to the $3^{\prime}$ coding sequence of PACS- 1 were amplified by PCR using mouse brain cDNA (generous gift from Dr. Donna Martin, University of Michigan, Ann Arbor, MI) and cloned into pGem-T Easy. Probes were generated using digoxigenin-labeling kit (Roche Applied Science), according to the manufacturer's protocol, and detected using alkaline phosphataseconjugated anti-digoxigenin antibodies with 5-bromo-4-chloro-3'indolyl phosphate p-toluidine salt and nitro-blue tetrazolium chloride.

Immunoprecipitations. Protein $\mathrm{G}$ agarose beads were preincubated with antibodies for $2-4 \mathrm{~h}$ on a nutator at $4^{\circ} \mathrm{C}$ in immunoprecipitation (IP) buffer containing the following: $20 \mathrm{~mm}$ Tris- $\mathrm{HCl}, \mathrm{pH} 7.5,1 \%$ Triton X-100, 25 mм NaF, $12.5 \mathrm{~mm} \mathrm{Na}_{4} \mathrm{P}_{2} \mathrm{O}_{7}, 0.1$ mм EDTA, $100 \mathrm{~mm} \mathrm{NaCl}, 2$ $\mathrm{mm} \mathrm{Na}_{3} \mathrm{VO}_{4}$, and protease inhibitors with $1 \%$ Triton X-100. Twentyfour hours after transfection, HEK293 cells were lysed on ice for $30 \mathrm{~min}$ in $500 \mu \mathrm{l}$ of IP buffer. After douncing, cells were centrifuged at $1000 \times \mathrm{g}$ for $5 \mathrm{~min}$, and supernatant was incubated overnight at $4^{\circ} \mathrm{C}$ with antibody-conjugated beads. Five percent of supernatant was used for starting material. Beads were washed three times for $5 \mathrm{~min}$ with IP buffer with $1 \%$ Triton X-100 and once with IP buffer without Triton X-100. Supernatant was either analyzed by SDS-PAGE as described previously (Jenkins et al., 2006), or used for in vitro kinase reactions.

In vitro kinase reactions. Kinase reactions were performed essentially as described previously (Boehning et al., 2003). Briefly, immunoprecipitates bound to beads were incubated with recombinant CK2 (New England Biolabs) in the presence of $10 \mu \mathrm{Ci} / \mathrm{ml}\left[\gamma^{-}{ }^{32} \mathrm{P}\right] \mathrm{ATP}$ and $200 \mu \mathrm{M}$ MgATP for $30 \mathrm{~min}$ at $30^{\circ} \mathrm{C}$ according to the manufacturer's protocol. Samples were processed by SDS-PAGE, and gels were exposed to Phos- 




E

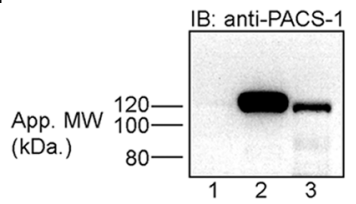

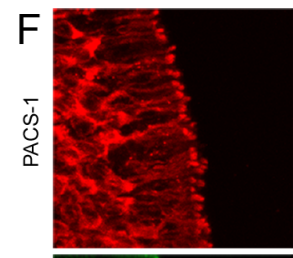
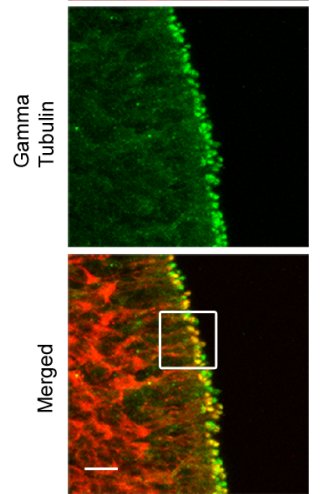


Figure 1. PACS-1 is expressed in 0SNs. A-D, In situ hybridizations performed on coronal sections from p1.5 CD1 mice were probed with digoxigenin-labeled antisense riboprobes for PACS-1 $(\boldsymbol{A}, \boldsymbol{C})$ or control, sense probes $(\boldsymbol{B}, \boldsymbol{D})$. PACS-1 mRNA is present in 0SNs as indicated by dark purple precipitate $(\boldsymbol{C})$. Line denotes transition from olfactory epithelium (top) to respiratory epithelium (bottom). Boxes in $\boldsymbol{A}$ and $\boldsymbol{B}$ represent regions of interest shown at higher magnification in $\boldsymbol{C}$ and $\boldsymbol{D}$, respectively. $\boldsymbol{E}$, Western blot results from lysates of untransfected HEK293 cells (lane 1), HEK293 cells expressing human PACS-1 with HA tag (lane 2), or p18 mouse olfactory epithelium (lane 3). Molecular weight markers are indicated on the left in kDa. $\boldsymbol{F}$, Immunostaining of coronal sections from adult CD1 mouse 0E demonstrates PACS-1 (red) is expressed in OSNs where it is enriched at the dendritic knob as indicated by colocalization with $\gamma$ tubulin (green). A merged image is shown on the bottom. Scale bar, $10 \mu \mathrm{m}$. $\mathbf{G}$, Higher-magnification image from boxed region of interest in $\boldsymbol{F}$ (bottom), demonstrating colocalization of PACS-1 and $\gamma$ tubulin.

phorImager screen for $1 \mathrm{~h}$ and read on a Typhoon Variable Mode Imager (GE Healthcare).

RNAi and retrovirus. Short-hairpin RNA for PACS-1 ( $5^{\prime}$ - sense GAT GAC AGC TTG ACT GAA ACA loop TTC AAG AGA antisense TGT TTC AGT CAA GCT GTC ATC-3') or Clontech negative control shRNA sequence was cloned into pSiren RetroQ (Clontech), and retrovirus was generated according to the manufacturer's protocol. Stable MDCK cell lines were created by infection with shRNA retrovirus followed by selection using $5 \mu \mathrm{g} / \mathrm{ml}$ puromycin.

Electroolfactograms. Summated potentials from mouse OE were recorded essentially as described previously (McEwen et al., 2007). Briefly, adult mice were killed by carbon dioxide inhalation, and the head was bisected laterally at the midline. Solutions were added for 5 min directly to the epithelial surface (Ringer's or Ringer's $+20 \mu \mathrm{M} \mathrm{TBB}$ ). Recording electrodes were placed on either side of the head in the region of turbinate II or IIb, and electrical responses to odorant (amyl acetate at $10 \mathrm{~mm}$ ) perfused across the $\mathrm{OE}$ in the vapor phase in a stream of humidified oxygen were recorded. We analyzed data with Clampfit (Molecular Devices) and determined peak heights from prepulse baseline.

\section{Results}

PACS-1 is expressed in olfactory sensory neurons

To address the question of whether PACS-1 regulates ciliary trafficking of the olfactory CNG channel, we first examined expression of PACS-1 in native olfactory tissue. Using in situ hybridization performed with an antisense PACS-1 riboprobe on coronal sections from multiple mice, we found that PACS-1 mRNA, indicated by a dark purple precipitate, is present along the dorsal roof of the nasal cavity in a region corresponding to the OSN layer (Fig. 1 $A, C$ ). Using a control, sense riboprobe, we detected minimal reactivity (Fig. $1 B, D$ ). These results clearly demonstrate the cellular distribution of PACS-1 mRNA in the olfactory epithelium and suggest that OSNs have the capacity to generate PACS-1 protein.

To determine whether PACS-1 protein is expressed in OE, we performed Western blot analysis of native olfactory tissue homogenate. Using antibodies directed against PACS-1, we de- tected the presence of an immunoreactive band corresponding to the molecular weight of PACS-1 $(\sim 120 \mathrm{kDa})$ in adult mouse OE (Fig. $1 E$, lane 3 ). The specificity of this signal was confirmed in that we detected a band of similar molecular weight (Fig. $1 E$, lane 2) using lysates from HEK-293 cells transiently transfected with hemagglutinin (HA)-tagged PACS-1 but not untransfected HEK-293 cells (Fig. 1 E, lane 1). Using antibodies against the HA epitope, we verified that the $120 \mathrm{kDa}$ band in lane 2 corresponds to PACS-1 (data not shown).

Since the OE comprises multiple cell types, including OSNs, sustentacular cells, and basal cells, we examined protein localization at the level of the OSN by immunostaining coronal sections of mouse OE. Single confocal planes of sections coimmunostained with antibodies against PACS-1 and $\gamma$ tubulin demonstrated the presence of PACS-1 protein in the OSN (Fig. $1 F, G$ ). Interestingly, PACS-1 localized to the dendritic knob, where it colocalized with the basal body marker, $\gamma$ tubulin, but was undetectable in olfactory cilia. Together, these results demonstrate that PACS-1 is expressed in OSNs and localizes to the dendritic knob, where it may act as part of the basal body protein complex to regulate ciliary transport of the CNG channel.

\section{The CNGB1b subunit can interact with PACS-1 and serve as a substrate for CK2 phosphorylation}

Previously, we have shown that the CNGB1b subunit is necessary for ciliary targeting of the olfactory CNG channel (Jenkins et al., 2006); however, the mechanism of this regulation remained unknown. The intracellular sorting protein PACS-1 interacts with its cargo through phosphorylated serines or threonines contained in a cluster of acidic residues on the cargo protein to control its subcellular localization. Interestingly, using amino acid sequence analysis of the three olfactory CNG channel subunits, we identified two highly conserved acidic clusters in the $\mathrm{N}$ terminus of the CNGB1b subunit that contain putative CK2 (formerly known as casein kinase II) consensus phosphorylation sites (Fig. 


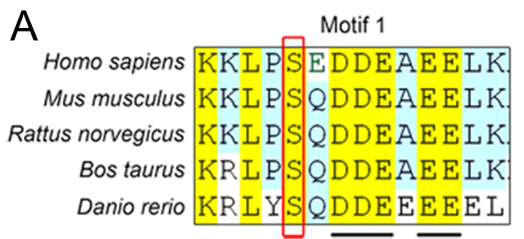

B
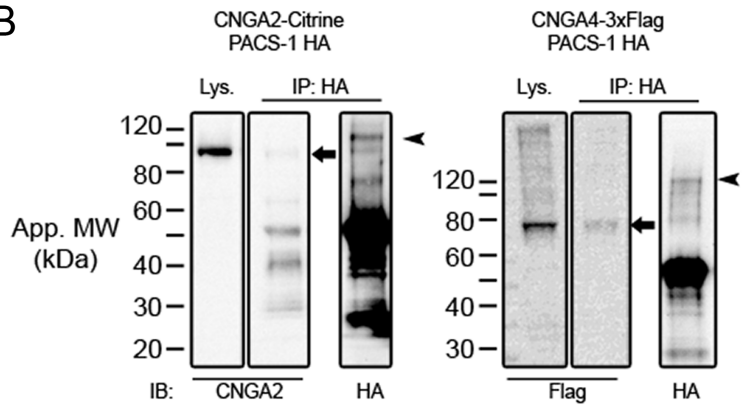

C



IB: PACS-1

D

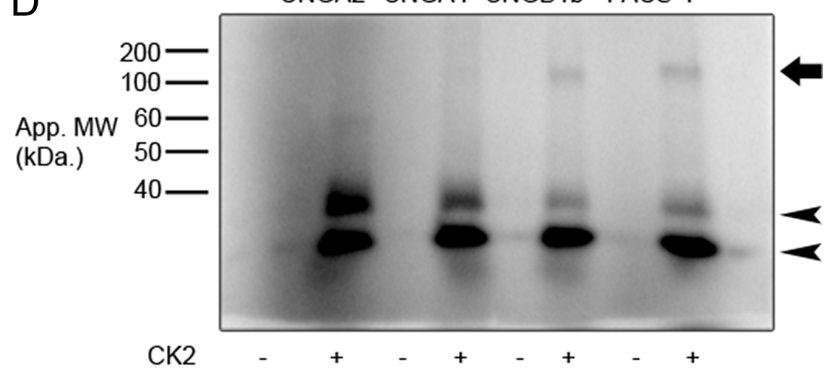

E

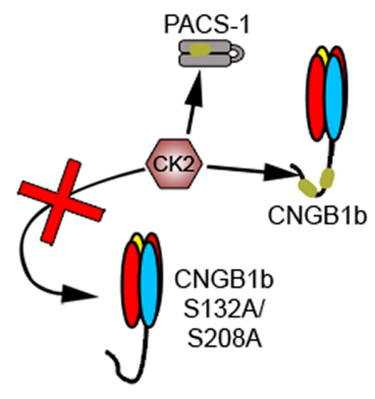

Figure 2. CNGB1b contains acidic clusters, interacts with PACS-1, and can serve as a substrate for CK2.A, Amino acid sequence alignment of the CNGB1b subunit from Homo sapiens, Mus musculus, Rattus norvegicus, Bos taurus, and Danio rerio. Acidic clusters marked by underline and putative CK2 phosphorylation sites marked by red rectangle. $B$, Immunoprecipitation experiments from HEK293 cells cotransfected with HA-PACS-1 and either CNGA2-mCitrine (top left), CNGA4-3xFlag (top right), or CNGB1b-3xFlag (bottom). Immunoprecipitations were performed with either rabbit anti-HA (IP: HA; top left, top right, bottom left) or rabbit anti-flag (IP: Flag; bottom right), and blots were probed with mouse CNGA2 (IB: CNGA2), mouse anti-Flag M2 (IB: Flag), or rabbit anti-HA (IB: HA). Arrows mark CNGA2 (top left), CNGA4 (top right), and CNGB1b (bottom left). Arrowheads mark PACS-1. Lys., lysates. C, Immunoprecipitation experiment from adult CD1 mouse olfactory epithelial lysates. Immunoprecipitations of endogenous protein were performed either with anti-CNGA2 or negative control, anti-HA antibodies. The blot was probed with anti-PACS-1 antibodies. The arrow marks PACS-1 protein at $\sim 120 \mathrm{kDa}$. SM, $10 \%$ starting material. $\boldsymbol{D}$, Autoradiograph from in vitro kinase reaction performed on immunoprecipitated proteins (CNGA2, CNGA4, CNGB1b, or PACS-1) in the presence $(+)$ or absence $(-)$ of recombinant CK2. Autophosphorylated CK2 bands are marked with arrowheads. The arrow marks the approximate molecular weight of PACS-1 and CNGB1b. E, Model depicting predicted CK2-mediated phosphorylation of both PACS-1 and CNGB1b, but not CNGB1b S132A/S208A.

$2 A)$. In contrast, no consensus PACS-1 binding sites were identified in CNGA2 or CNGA4.

To examine interactions between PACS-1 and CNG channel subunits, we used immunoprecipitation experiments from both transfected cells and native olfactory tissue. Immunoprecipitations of CNGB1b from HEK293 cells transiently transfected with CNGB1b-3xFlag and HA-PACS-1 demonstrated an in vitro interaction that was confirmed using the reverse immunoprecipitation (Fig. $2 \mathrm{~B}$, bottom). Importantly, immunoprecipitations of PACS-1 from HEK293 cells transiently transfected with either CNGA2-Citrine or CNGA4-3xFlag demonstrated only weak binding between PACS-1 and the channel subunits (Fig. 2 B, top), thus confirming the specificity of the interaction between PACS-1 and CNGB1b. This is consistent with previous reports showing only weak interaction of PACS-1 with proteins lacking acidic clusters (Piguet et al., 2000). Importantly, we also observed an interaction between the endogenous CNG channel and PACS-1 in native mouse olfactory epithelium, while negative control antibodies showed no detectable immunoprecipitation (Fig. 2C). Although we performed these experiments with antibodies against CNGA2 due to lack of suitable immunoprecipitation antibodies against CNGB1b, immunoisolation of CNGA2 has been shown to efficiently isolate the entire native CNG chan- nel complex, including CNGA4 and CNGB1b (Jenkins et al., 2006; Michalakis et al., 2006). Together these results indicate that CNGB1b and PACS-1 can interact in complex both in vitro and in vivo.

We next tested whether the CNGB1b subunit could serve as a substrate for CK2 phosphorylation. In vitro kinase reactions were performed on immunoprecipitated protein (CNGA2, CNGA4, CNGB1b, or PACS-1) from transiently transfected HEK293 cells using recombinant CK2 and $\left[\gamma^{-}{ }^{32} \mathrm{P}\right] \mathrm{ATP}$. As predicted from the sequence analysis, we found that CNGB1b serves as a substrate for CK2 (Fig. 2D), while PACS-1 was also phosphorylated by CK2 as previously described (Scott et al., 2003). In contrast, despite efficient immunoprecipitation (supplemental Fig. 1, available at www.jneurosci.org as supplemental material), neither CNGA2 nor CNGA4 were phosphorylated (Fig. 2D), demonstrating a subunit specificity for CK2 phosphorylation.

Finally, we used alanine substitution to determine whether the putative CK2 sites at serines 132 and 208 within CNGB1b are capable of being phosphorylated by the enzyme. For these experiments, we created three mutant forms of CNGB1b (S132A, S208A, and S132A/S208A double mutant) and performed in vitro kinase reactions on immunoprecipitated proteins. Despite equal immunoprecipitation, the CNGB1b S132A/S208A mutant ex- 
A
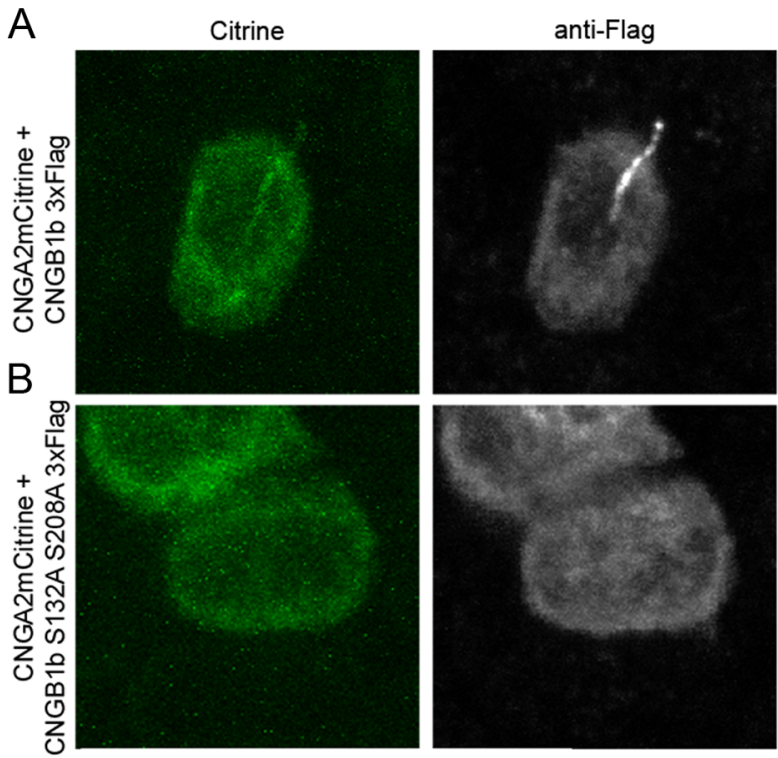

C


Figure 3. Mutation of CK2 phosphorylation sites on the $N$ terminus of CNGB1b impairs ciliary trafficking of the CNG channel. $\boldsymbol{A}, \boldsymbol{B}$, Representative confocal images of MDCK cells transfected with CNGA2-mCitrine and CNGB1b-3xFlag ( $\boldsymbol{A}$ ) or CNGB1b S132A, S208A$3 x F l a g(B)$. Citrine signal is on the left (green). Flag immunostaining for CNGB1b is in the middle (grayscale). Merged image with staining for acetylated $\alpha$ tubulin (red) is on the right. Scale bars, $10 \mu \mathrm{m}$. $\boldsymbol{C}, \boldsymbol{D}$, Average data from multiple cells ( $n=6-7)$ for CNGB1b wild-type ( $\boldsymbol{C}$ ) or CNGB1b S132A, S208A (D). Data were apportioned into bins of 1/10 of normalized cilia length. Acetylated tubulin signal is shown in red, CNGA2-mCitrine fluorescence in green, and anti-Flag immunostaining signal in blue (arbitrary fluorescent units).

or S208 alone caused an intermediate decrease in ciliary trafficking of the channel (supplemental Fig. 2, available at www. jneurosci.org as supplemental material). Together these data indicate that, while either CK2 site is sufficient for ciliary trafficking, both sites are necessary for ciliary enrichment of the CNG channel.

\section{Loss of PACS-1 function impairs CNG channel ciliary localization}

To ascertain a functional role for PACS-1 in the ciliary targeting of the olfactory CNG channel, we generated retroviral stable MDCK cells expressing either wildtype PACS-1 or the dominant-negative, nonphosphorylatable PACS-1 S278A mutant. It is thought that phosphorylation of an autoregulatory domain of PACS-1 at serine 278 causes the protein to unfold, allowing the binding and subsequent trafficking of cargo protein. This phosphorylation-dependent function is inhibited by mutation of S278 to alanine (Scott et al., 2003). Expression of CNGA2mCitrine and CNGB1b in PACS-1 S278A stable MDCK cells resulted in impaired ciliary localization, whereas in cells stably overexpressing wild-type PACS-1 the channel localized to cilia as expected (Fig. 4A, B; supplemental Fig. 3, available at www.jneurosci.org as supplemental material). Importantly, the inhibition of channel ciliary targeting in the presence of PACS-1 S278A was also seen with channels containing CNGA2, CNGA4, and CNGB1b as in the native complex (supplemental Fig. 3C, available at www.

hibited an $\sim 35 \%$ decrease in phosphorylation by CK2 compared with wild-type $(66.44 \pm 1.958 \%$ compared with CNGB1b wildtype; $n=3$ experiments, $p<0.0001$ unpaired $t$ test). Mutation of either serine alone (S132A or S208A) caused an intermediate decrease in phosphorylation (data not shown). These results demonstrate that CK2 is capable of phosphorylating S132 and S208 located within acidic clusters on CNGB1b, which may serve to regulate CNGB1b ciliary trafficking.

\section{Mutation of the CK2 phosphorylation sites on CNGB1b inhibits ciliary delivery of the CNG channel}

Since mutation of CK2 phosphorylation sites within PACS-1 cargo has been shown to impair intracellular trafficking (Jones et al., 1995), we tested CNG channel trafficking using phosphorylationdeficient CNGB1b mutants. Previously, we have demonstrated that ciliated MDCK cells represent a suitable model to study ciliary trafficking of olfactory signaling proteins (Jenkins et al., 2006). Confocal images of individual cells clearly show that mutation of both putative CK2 sites (S132A + S208A double mutant) caused a dramatic loss of CNGB1b and its heteromeric partner CNGA2mCitrine from the cilia of MDCK cells (Fig. $3 A, B$ ) (supplemental Fig. 3, available at www.jneurosci.org as supplemental material). Importantly, averaged data from multiple cells indicate an almost complete absence of CNG channel ciliary localization in the presence of the CNGB1b double mutant (Fig. 3C,D). Mutation of either S132 jneurosci.org as supplemental material). As an additional control, MDCK cells stably expressing the phosphomimetic form of PACS-1 (PACS-1 S278D) demonstrated normal ciliary localization of the CNG channel (data not shown). These data indicate that loss of the autoregulatory phosphorylation site on PACS-1 inhibits ciliary trafficking of the CNG channel.

One limitation of using the nonphosphorylatable mutant PACS- 1 is that its overexpression may sequester trafficking partners within the cell, leading to nonspecific effects. To overcome this limitation, we used retrovirally delivered short hairpin RNA (shRNA) in MDCK cells to silence PACS-1 expression. MDCK cells express endogenous PACS-1, which was efficiently silenced using a $21 \mathrm{nt}$ sequence directed against PACS-1 (human 13451365), while a validated negative control shRNA had no effect on PACS-1 protein levels (supplemental Fig. 4, available at www. jneurosci.org as supplemental material). In MDCK cells stably expressing PACS-1 shRNA, we failed to detect localization of CNGA2-mCitrine to cilia in the presence of CNGB1b, whereas, in cells stably expressing negative control shRNA, we found normal ciliary localization of the CNG channel (Fig. 4C,D). This lack of ciliary localization seen in the PACS-1 shRNA stable cells was not due to the absence of the CNGB1b subunit (supplemental Fig. $3 D$, available at www.jneurosci.org as supplemental material). Interestingly, mutation or shRNA-mediated suppression of PACS-1 had no effect on cilia length or gross morphology (sup- 

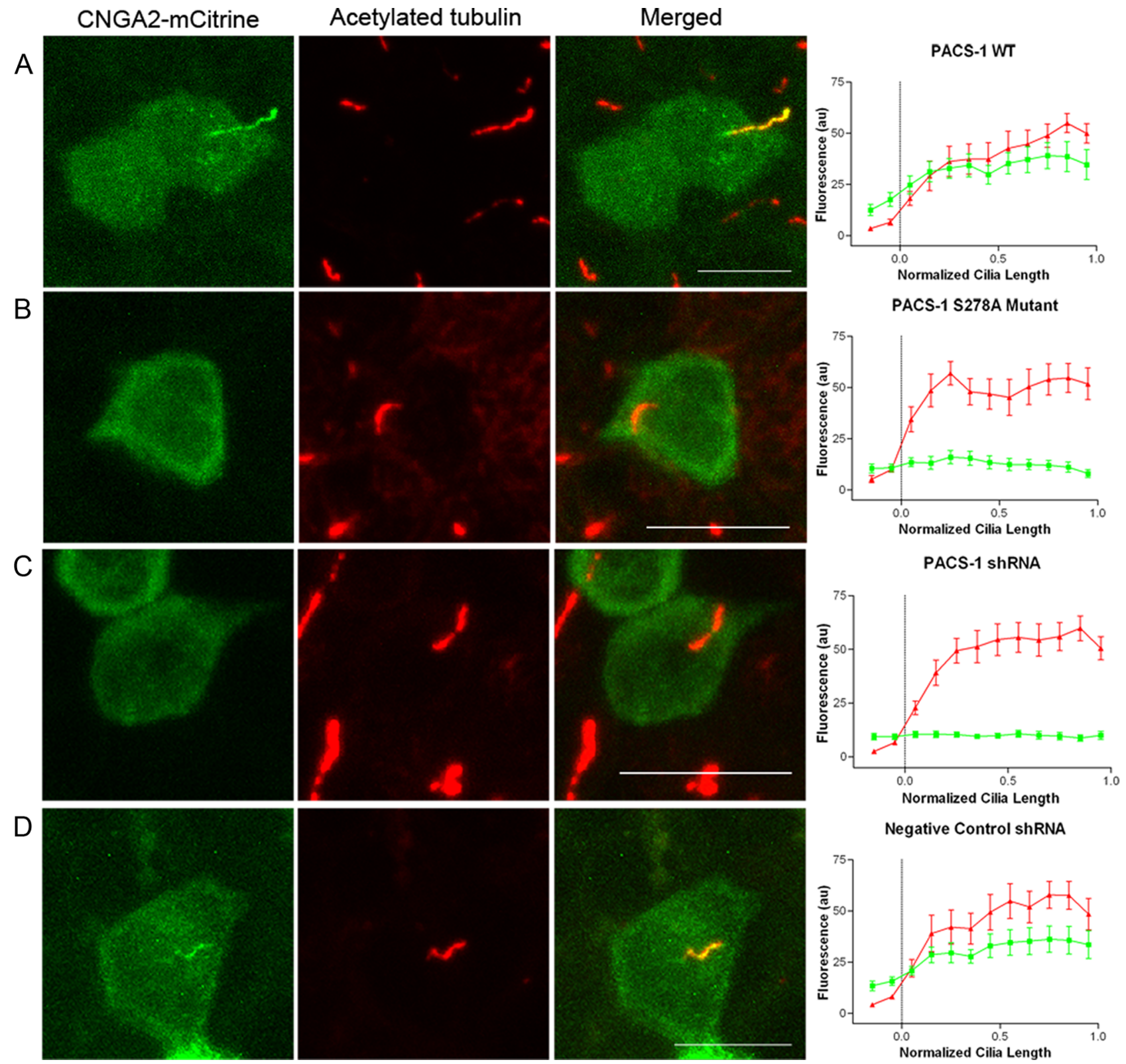

Figure 4. PACS-1 regulates ciliary trafficking of the olfactory CNG channel. $A-D$, CNGA2-mCitrine and CNGB1b-3xFlag were transfected into MDCKstable cell lines expressing wild-type PACS-1 (A), PACS-1 S278A nonphosphorylatable mutant (B), shRNA directed against PACS-1 ( $)$, or negative control shRNA (D). Signal from CNGA2-mCitrine is shown on the left (green) and acetylated tubulin signal marking the ciliary axoneme is in the middle (red), with the merged image on the right. Scale bars, $10 \mu \mathrm{m}$. Average data from multiple cells $(n=9-11$ for each condition) are shown on the far right. Data were apportioned into bins of 1/10 of normalized cilia length. Acetylated tubulin signal shown in red and CNGA2-mCitrine fluorescence in green (arbitrary fluorescent units).

plemental Fig. 4, available at www.jneurosci.org as supplemental material). These results, together with the results from the nonphosphorylatable mutant PACS-1, indicate that diminished PACS-1 function, either by mutation of the autoregulatory domain or loss of protein, leads to impaired ciliary trafficking of the olfactory CNG channel.

\section{Inhibition of CK2 alters CNG channel localization}

Pharmacological inhibition of CK2 enzymatic activity has been shown to impair PACS-1 activity (Jones et al., 1995; Scott et al., 2003, 2006a). To directly examine the CK2 phosphorylation dependence of ciliary trafficking of the olfactory CNG channel, we transfected MDCK cells with both CNGA2-mCitrine and CNGB1b, allowed $48 \mathrm{~h}$ for channel to reach cilia, and then treated cells with the cell-permeant, selective CK2 inhibitor, TBB. Inhi- bition of CK2 for $4 \mathrm{~h}$ with $20 \mu \mathrm{M}$ TBB led to a loss of CNG channel from the cilia of MDCK cells (Fig. 5A). Mislocalized channel formed bright puncta at the base of the cilium, which can be seen more clearly in an $X-Z$ projection (Fig. $5 B$ ). Consistent with the effects of phosphodeficient PACS-1 shown above, treatment of MDCK cells with $20 \mu \mathrm{M}$ TBB for $4 \mathrm{~h}$ had no effect on average cilia length (supplemental Fig. 5, available at www.jneurosci.org as supplemental material). Together, these indicate that the processes necessary for maintenance of cilia length are not dependent on CK2 phosphorylation.

CK2 phosphorylation is necessary for the ciliary localization of CNG channel in vivo and proper olfactory function

Our results indicate an important role for PACS-1 and CK2 phosphorylation in the ciliary trafficking of ectopically expressed 


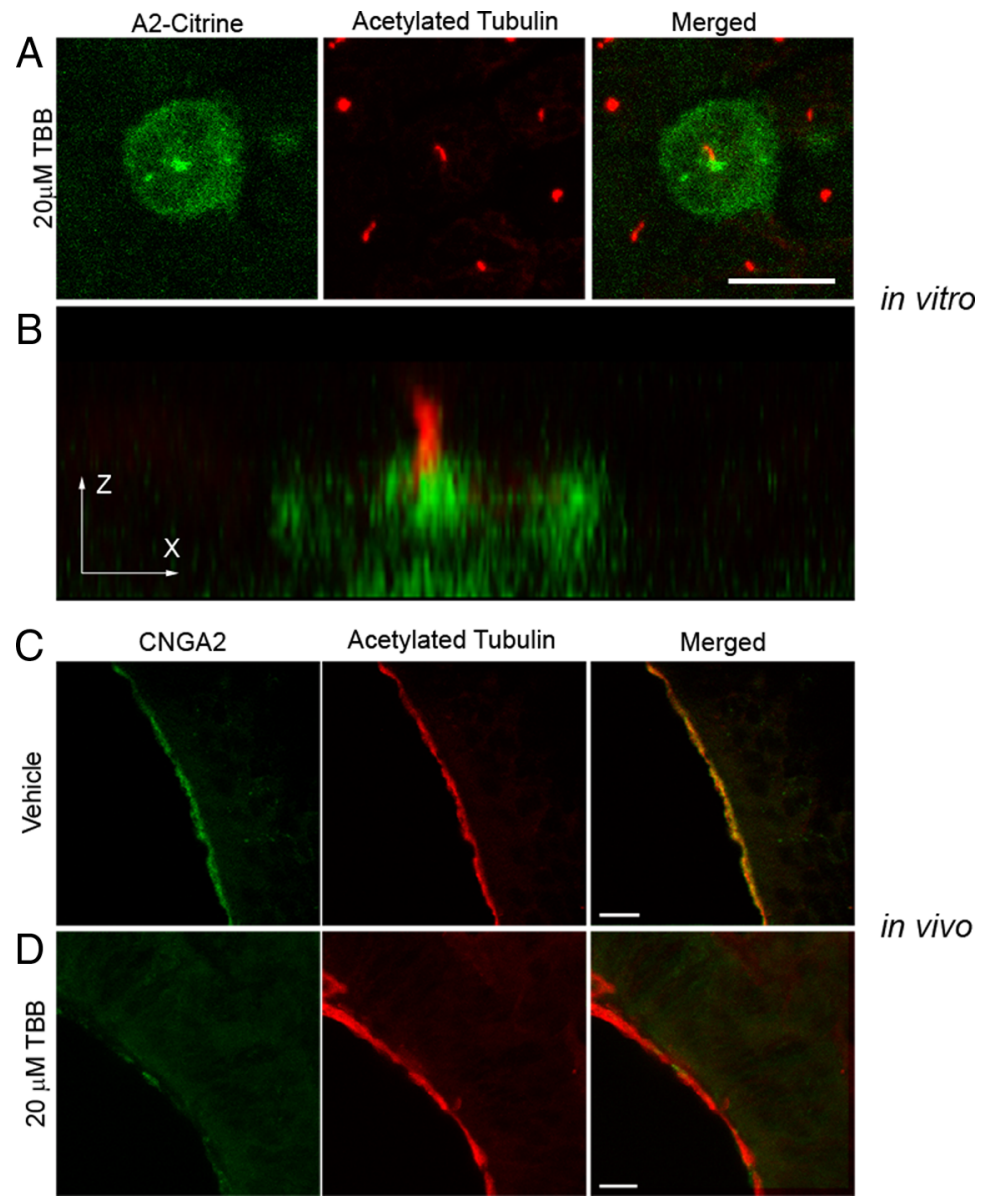

Figure 5. Inhibition of CK2 activity causes a loss of CNG channel ciliary localization. $A, B, M D C K$ cells were transfected with CNGA2-mCitrine and CNGB1b-3xFlag and allowed time for ciliary delivery followed by $4 \mathrm{~h}$ of treatment with a $20 \mu \mathrm{m}$ concentration of the CK2 inhibitor, TBB. A, Compressed confocal image of a MDCK cell with CNGA2-mCitrine fluorescence shown in green and acetylated tubulin staining marking cilia shown in red. Scale bar, $10 \mu \mathrm{m} . \boldsymbol{B}, X-Z$ projection of the cell from $\boldsymbol{A}$ demonstrating concentration of CNG channel at base of cilium. $C, D$, Representative compressed confocal stacks of coronal sections from 0 E from intranasally injected CD1 mice. Confocal images of OE from adult CD1 mice treated with DMSO vehicle $(C)$ or $20 \mu \mathrm{m} \mathrm{TBB} \mathrm{(D)} \mathrm{for} 4 \mathrm{~h}$. Signals shown are CNGA2 immunostaining (green, left), and acetylated tubulin immunostaining (red, middle). Merged images are shown on the right. Scale bars, $10 \mu \mathrm{m}$.

CNG channels in vitro. We next tested the hypothesis that these mechanisms were operating in native olfactory tissue and were necessary for proper olfactory function. To this end, the selective CK2 inhibitor TBB was delivered directly to the olfactory epithelium using intranasal injections into adult CD1 mice. Using this method, we found that the area of greatest exposure to the inhibitor were regions of the ventral OE, especially turbinates III and IV. This was confirmed using the fluorescent tracer, carboxyfluorescein diacetate, succinimidyl ester (data not shown). Fluorescence immunohistochemistry on fixed tissue was used to examine the subcellular distribution of CNG channels. Coronal sections from mice injected with control solution (0.1\% DMSO) exhibited normal CNG channel enrichment in cilia, as indicated by colocalization with the ciliary marker, acetylated $\alpha$ tubulin (Fig. 5C). Sections from mice injected with $20 \mu \mathrm{M}$ TBB followed by $4 \mathrm{~h}$ of recovery demonstrated a dramatic loss of CNG channel from the cilia layer and a lower level of total CNG channel protein in the olfactory epithelium (Fig. 5D). This loss of channel protein is consistent with previous work demonstrating that mislocalized CNG channel out of the cilia is degraded in OSNs (Michalakis et al., 2006). These results demonstrate that the ciliary trafficking of the CNG channel in native olfactory tissue is dependent on CK2 phosphorylation.

Mislocalization of olfactory signaling proteins from cilia leads to olfactory dysfunction (Michalakis et al., 2006; McEwen et al., 2007). To assess the effect of CK2 inhibition on olfactory function we measured the odor-evoked electrical response in OSNs using electro-olfactogram (EOG) recordings from the surface of mouse $\mathrm{OE}$. The head was split sagittally at the midline to expose the olfactory turbinates. Solutions (Ringer's buffer control or Ringer's plus $20 \mu \mathrm{M}$ TBB) were applied directly to either turbinate and evoked potentials in response to the odorant amyl acetate were measured every $5 \mathrm{~min}$ from two recording electrodes placed on opposite sides of the $\mathrm{OE}$ (see diagram in Fig. 6). In paired recordings from five mice, inhibition of CK2 with TBB caused a significant and almost complete loss of the response to amyl acetate within 25 min. Treatment with control solution caused only a slight decrease (Fig. 6A,B) consistent with rundown of the odor-evoked signal (Scott et al., 2006b). To confirm that the mechanism for the loss of EOG response was due to an alteration in the ciliary localization of the CNG channel, we treated mouse $\mathrm{OE}$ with TBB or control solution for $25 \mathrm{~min}$ in the same manner as performed for EOG recordings. Medial surfaces of the olfactory turbinates, which correspond to regions of the $\mathrm{OE}$ that would have been exposed to drug, demonstrated a dramatic decrease in ciliary localization of the $\mathrm{CNG}$ channel compared with control (Fig. 6C). After only $25 \mathrm{~min}$, mislocalized channel protein appeared to accumulate on $\mathrm{mi}$ crotubule tracks of the dendrite, indicating a possible retrograde trafficking of the channel in response to CK2 inhibition. Interestingly, ACIII ciliary localization was unaffected by the 25 min CK2 inhibition (supplemental Fig. 5, available at www.jneurosci.org as supplemental material). These data demonstrate that inhibition of CK2 causes a selective mislocalization of olfactory CNG channels from cilia and subsequent olfactory dysfunction.

\section{Adenoviral expression of nonphosphorylatable PACS-1 in native OSNs impairs ciliary localization of the endogenous CNG channel}

Since inhibition of CK2 in native OSNs causes a selective mislocalization of the CNG channel from cilia, we examined whether this effect included inhibition of PACS-1 function. Mice were infected intranasally with adenovirus carrying either nonphosphorylatable PACS-1 S278A IRES GFP or GFP alone. Infection of OSNs with adenovirus carrying the nonphosphorylatable form of PACS-1 (S278A) caused a mislocalization of the endogenous ciliary CNG channel, but no detectable change in the cilia layer as indicated by acetylated $\alpha$ tubulin staining (Fig. 7A, top). Expression of PACS-1 S278A, however, had no effect on ciliary ACIII localization (Fig. $7 A$, bottom), consistent with the specificity 
measured by pharmacological inhibition of CK2 (supplemental Fig. 5B, available at www.jneurosci.org as supplemental material). A surprising result was the malformation of the dendrite in OSN infected with the functionally impaired S278A mutant. In these neurons, marked by GFP, there was an obvious swelling of the knob and thickening of the dendritic shaft compared with controls (Fig. 7A,B), however these cells were localized deep within the OMP-positive cell body layer as would be expected for mature OSNs (supplemental Fig. 6, available at www. jneurosci.org as supplemental material). This unanticipated result suggests that, although we observe no accumulation of CNG channel, inhibition of PACS-1mediated trafficking may cause the accumulation of other dendritic or ciliary cargo. As a control for adenovirusmediated cytotoxicity, we infected $\mathrm{OE}$ with adenovirus expressing only GFP, and found no change in OSN morphology or CNG channel ciliary localization (Fig. 7B). In addition, previous work has shown that adenovirus can be used for gene delivery into OSNs, which continue to demonstrate normal morphology and function after infection (Ivic et al., 2000; Youngentob et al., 2004). Although we have ruled out adenovirus-mediated cytotoxicity, we cannot exclude the possibility that PACS-1 S278A overexpression is causing cellular degradation and differential mislocalization of CNG channel versus ACIII. To examine specificity of the effect of alterations in PACS-1 function on CNG channel we examined the ciliary localization of ACIII-GFP in MDCK cells, which can tolerate long-term depletion of PACS-1 or overexpression of mutant PACS-1 with no noticeable changes in cilia or cellular survival (supplemental Fig. 7, available at www.jneurosci.org as supplemental material). In these cells, we found that expression of PACS-1 S278A or PACS-1 shRNA had no effect on ACIII-GFP ciliary localization compared with controls (supplemental Fig. 7, available at www.jneurosci.org as supplemental material). These results demonstrate that PACS-1 specifically affects ciliary localization of the CNG channel, but not ACIII, in native OSNs.

\section{Discussion}

The results of these studies define a mechanism for the subunit-dependent ciliary trafficking of the olfactory CNG channel. The intracellular sorting protein, PACS-1, is expressed in OSNs and localizes to the dendritic knob, which is the site of protein entry and exit from

A

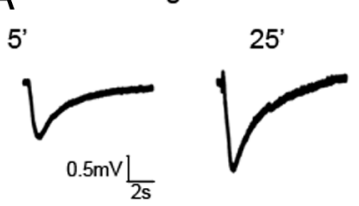

$20 \mu \mathrm{M}$ TBB
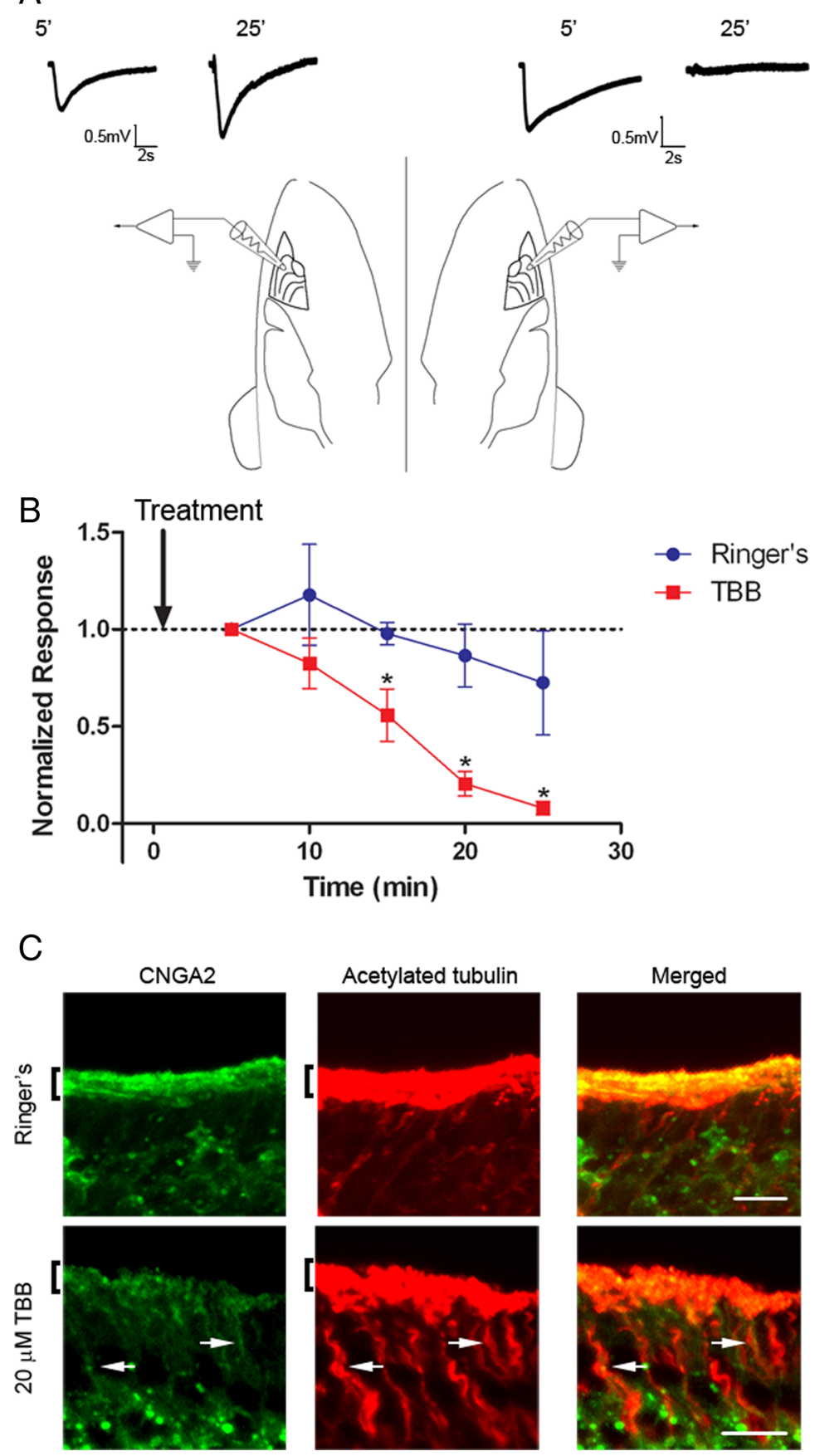

Figure 6. Inhibition of CK2 alters the ciliary localization of olfactory signaling proteins. CK2 inhibition impairs olfactory function. $A$, Representative traces from paired $E 0 G$ recordings from turbinate II or Ilb in response to amyl acetate. Mice were prepared as indicated in Materials and Methods, and two electrodes were placed on the surface of the OE on opposite sides of the head. OE was treated with Ringer's solution control (left) or Ringer's plus $20 \mu \mathrm{M}$ TBB (right) for 5 min and then EOG responses were recorded every $5 \mathrm{~min}$. Representative traces are shown at 5 and $25 \mathrm{~min}$ for both treatments. Calibration: 0.5 $\mathrm{mV}, 2$ s. $\boldsymbol{B}$, Average data demonstrate a rapid loss of EOG response from the TBB-treated OE $(n=5)\left({ }^{*} p<0.05\right.$; paired $t$ test). Responses were normalized to the first trace taken at 5 min after treatment. The arrow indicates time of drug delivery. C, Representative images taken from the medial surface of the olfactory turbinates exposed to either Ringer's control (top) or $20 \mu \mathrm{M}$ TBB (bottom) for 25 min. Sections were coimmunostained with antibodies against CNGA2 (left, green) and acetylated $\alpha$ tubulin (middle, red). Merged images shown on right. Scale bars, $10 \mu \mathrm{m}$. Arrows mark dendritic tracks with concentrated CNGA2 signal. Brackets denote cilia layer.

olfactory cilia. We discovered that PACS-1 binds to the CNGB1b subunit and controls its proper ciliary localization. This mechanism is dependent on CK2 phosphorylation at sites on both PACS-1 and two distinct sites on the $\mathrm{N}$ terminus of the CNGB1b 
A

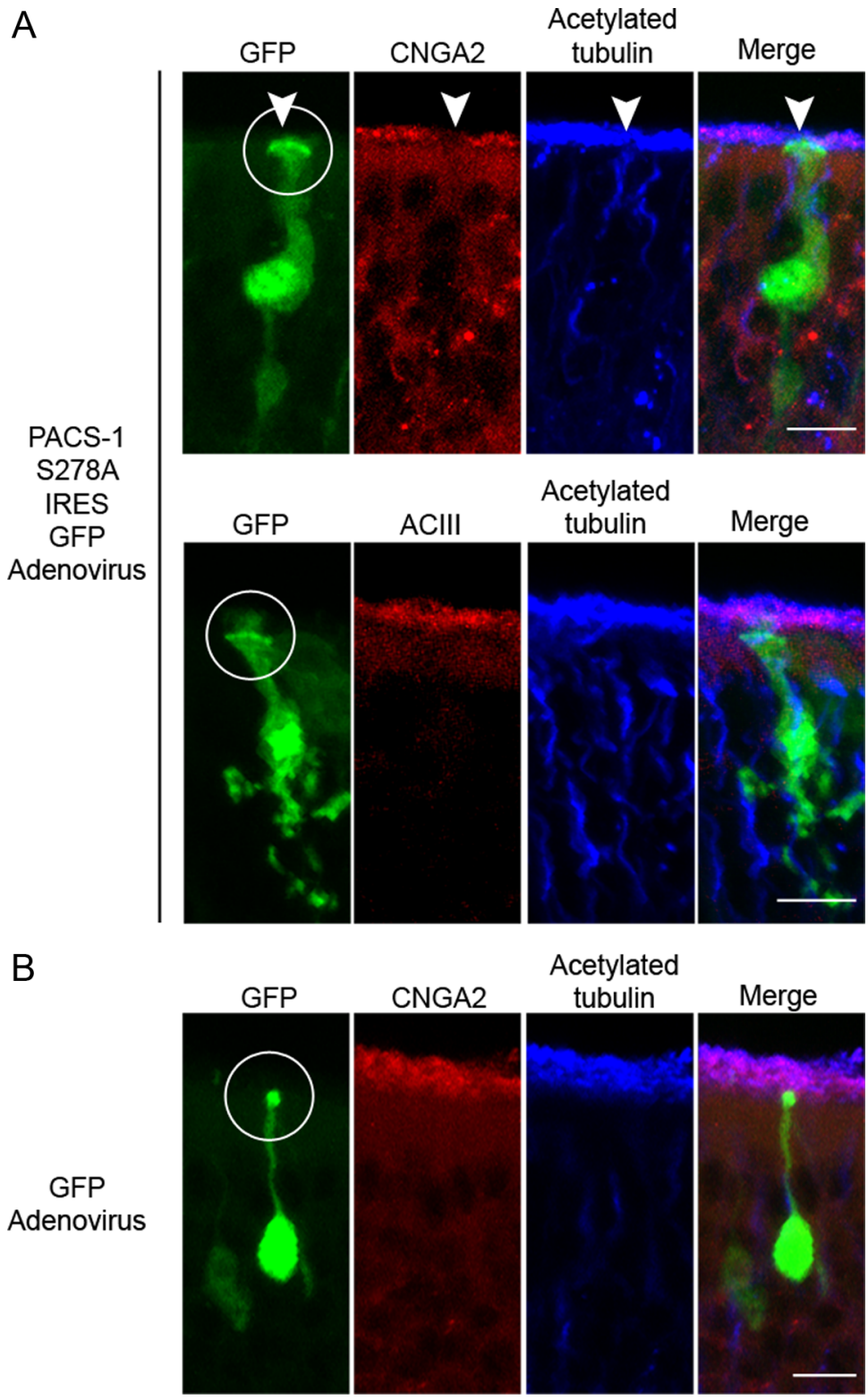

Figure 7. Expression of mutant PACS-1 in native OSNs causes mislocalization of the CNG channel, but not ACIII. Representative collapsed confocal images of coronal sections of $O E$ from adenovirally infected mice. $A$, Top, Immunostaining of a PACS-1 S278A IRES GFP-infected OSN (green) with antibodies against CNGA2 (red) and acetylated $\alpha$ tubulin (blue) demonstrates a loss of ciliary CNG channel from the infected OSN (white arrowhead) with no change in the cilia layer. Bottom, Immunostaining of a PACS-1 S278A IRES GFP-infected OSN (green) with antibodies against ACIII (red) and acetylated $\alpha$ tubulin (blue) demonstrates no detectable change in ciliary ACIII or the cilia layer from the infected OSN. $B$, Immunostaining of a GFP-infected OSN (green) with antibodies against CNGA2 (red) and acetylated $\alpha$ tubulin (blue) demonstrates no detectable change in ciliary CNG channel or the cilia layer from the infected OSN. For all conditions, a merged image is shown on the right (Merge), white circles mark dendritic knobs, and scale bars represent $10 \mu \mathrm{m}$. this mutant protein may lead to nonspecific effects through the sequestration of important binding partners. To overcome this limitation, we confirmed our dominant-negative results using shRNAmediated gene silencing of PACS- 1 to confirm its role in the ciliary localization of the olfactory CNG channel.

Although never directly addressed, there are other reports that are consistent with a role for PACS-1 in the trafficking of olfactory cargo. Some PACS-1 binding partners, such as the AP-1 adaptor complex, have been shown to be necessary for proper PACS-1-mediated trafficking through the budding of clathrin-coated vesicles from the trans-Golgi network (Crump et al., 2001). Interestingly, components of the Golgilocalized clathrin trafficking machinery as well as members of IFT complex, which participate in ciliary transport, share significant homology (Avidor-Reiss et al., 2004). In addition, the clathrin AP- $1 \mu$ adaptor UNC-101 has been shown to be responsible for the localization of odorant receptors to the cilia of Caenorhabditis elegans (Dwyer et al., 2001). These peripherally related reports may suggest that PACS-1 participates in a common transport pathway for all olfactory ciliary proteins. Our results in native olfactory tissue, however, indicate that the requirement of CK2 phosphorylation does not apply to all olfactory signaling proteins.

There has been controversy in the role of CK2 in the regulation of the subcellular localization of other ciliary ion channels. For example, recent work reports that inhibition of CK2, despite altering the subcellular localization of the TRP-channel polycystin-2, had no effect on ciliary expression in MDCK cells (Köttgen et al., 2005). In contrast, other work in C. elegans, demonstrated that a nonphosphorylatable mutant of polycystin-2 localizes to cilia whereas the phosphomimetic form of the protein fails to traffic to cilia (Hu et al., 2006). Additionally, work in zebrafish demonstrated that deletion of a region of polycystin-2 corresponding to an acidic cluster with a CK2 phosphorylation site causes mislocalization of polycystin-2 to the subunit. Importantly, this work provides new insight into the requirements for the ciliary trafficking of polytopic membrane proteins and demonstrates for the first time a functional role for PACS-1 in neurons.

This is the first report for the direct mechanistic role of PACS-1 in the ciliary trafficking of membrane proteins. Previously, a role for PACS-1 in the localization of nephrocystin to the ciliary base/transition zone in epithelial cells was reported (Schermer et al., 2005). This work relied solely on the expression of nonphosphorylatable dominant-negative constructs to implicate PACS-1 in the localization. However, overexpression of apical cell membrane (Obara et al., 2006). These findings may highlight differences in trafficking pathways for polycystin-2 and the CNG channel or differences between model systems. Interestingly, polycystin-2 and the CNGB1b subunit share a common "RVxP" trafficking motif, and mutation of this motif causes mislocalization of both proteins from cilia (Geng et al., 2006; Jenkins et al., 2006).

The dynamics of intraciliary movement and the time for entry and exit from the cilia compartment are areas of intense interest. Very little is known regarding the residence time of proteins, including ion channels, within the ciliary membrane. Our results demonstrate that inhibition of CK2 causes a near- 
complete loss of CNG channel from the cilia of OSNs in $\sim 25$ min. Additionally, as shown in Figure 6, upon inhibition of CK2, the CNG channel displays an increased localization along the microtubule tracks of the dendrites. This rapid loss of channel from cilia can result from inhibited entry or accelerated exit of channels from the ciliary compartment. This disruption of the steady-state channel localization implies a bidirectional movement of channel protein on a time scale shorter than $25 \mathrm{~min}$. Qin et al. previously demonstrated that the C. elegans TRPV channels OSM-9 and OCR-2 move bidirectionally along the ciliary axoneme at rates comparable to IFT $(\sim 1 \mu \mathrm{m} / \mathrm{s})$. If this applies to movement into and out of the cilium, it suggests a very short residence time for individual channels within the cilia. Our previous work demonstrated that the rate of olfactory CNG channel movement within MDCK cell cilia is on the order of diffusion with a $\tau$ of $\sim 10$ min (Jenkins et al., 2006), which predicts a greater latency for the channel. Therefore, we cannot exclude the possibility of an active cargo retrieval process initiated upon CK2 inhibition. The failure to detect PACS-1 in the cilia of OSNs (Fig. 1), however, suggests that PACS-1 is not the protein responsible for this potential scavenging mechanism. Ultimately, elucidation of the dynamics of protein movement into and out of the cilium will require advanced imaging techniques with the appropriate temporal and spatial resolution.

In summary, our results demonstrate that PACS-1 and CK2 are important players in the regulation of the localization of select olfactory cilia cargo. Chronic inhibition of PACS-1 function using dominant-negative constructs or shRNA-mediated gene silencing impairs the steady-state ciliary localization of the olfactory CNG channel in MDCK cells. Interestingly, expression of nonphosphorylatable PACS-1 in native OSNs, in addition to causing mislocalization of ciliary CNG channel, also leads to a dramatic thickening of the dendrite and flattening of the dendritic knob. These results may implicate PACS-1 in the trafficking of other dendritic or ciliary cargo. Interestingly, however, expression of this dominant-negative PACS-1 did not alter localization of ACIII, suggesting that PACS-1 controls the subcellular localization of only a subset of neuronal cargo. In contrast to the long-term, steady-state effects seen with PACS-1 dominantnegative expression, pharmacological inhibition of CK2 phosphorylation causes an acute and rapid mislocalization of the CNG channel, while also not affecting other members of the olfactory signaling cascade. Since activation of the CNG channel in cilia serves as the trigger for action potential generation in the OSN, CK2 phosphorylation-dependent trafficking may represent a novel mechanism for the fine tuning of responses to sensory stimuli. In addition to these physiological implications, our results clearly link PACS-1 to the subunit-dependent ciliary localization of the olfactory CNG channel and highlight a novel role for PACS-1 in the regulation of subcellular protein trafficking in neurons.

\section{References}

Avidor-Reiss T, Maer AM, Koundakjian E, Polyanovsky A, Keil T, Subramaniam S, Zuker CS (2004) Decoding cilia function: defining specialized genes required for compartmentalized cilia biogenesis. Cell 117:527-539.

Badano JL, Mitsuma N, Beales PL, Katsanis N (2006) The ciliopathies: an emerging class of human genetic disorders. Annu Rev Genomics Hum Genet 7:125-148.

Boehning D, Moon C, Sharma S, Hurt KJ, Hester LD, Ronnett GV, Shugar D, Snyder SH (2003) Carbon monoxide neurotransmission activated by CK2 phosphorylation of heme oxygenase-2. Neuron 40:129-137.
Christensen ST, Pedersen LB, Schneider L, Satir P (2007) Sensory cilia and integration of signal transduction in human health and disease. Traffic 8:97-109.

Crump CM, Xiang Y, Thomas L, Gu F, Austin C, Tooze SA, Thomas G (2001) PACS-1 binding to adaptors is required for acidic cluster motifmediated protein traffic. EMBO J 20:2191-2201.

Dwyer ND, Adler CE, Crump JG, L’Etoile ND, Bargmann CI (2001) Polarized dendritic transport and the AP-1 mul clathrin adaptor UNC101 localize odorant receptors to olfactory cilia. Neuron 31:277-287.

Fuchs JL, Schwark HD (2004) Neuronal primary cilia: a review. Cell Biol Int 28:111-118.

Geng L, Okuhara D, Yu Z, Tian X, Cai Y, Shibazaki S, Somlo S (2006) Polycystin-2 traffics to cilia independently of polycystin-1 by using an N-terminal RVxP motif. J Cell Sci 119:1383-1395.

Hu J, Bae YK, Knobel KM, Barr MM (2006) Casein kinase II and calcineurin modulate TRPP function and ciliary localization. Mol Biol Cell 17:2200-2211.

Hüttl S, Michalakis S, Seeliger M, Luo DG, Acar N, Geiger H, Hudl K, Mader R, Haverkamp S, Moser M, Pfeifer A, Gerstner A, Yau KW, Biel $M$ (2005) Impaired channel targeting and retinal degeneration in mice lacking the cyclic nucleotide-gated channel subunit CNGB1. J Neurosci 25:130-138.

Ivic L, Pyrski MM, Margolis JW, Richards LJ, Firestein S, Margolis FL (2000) Adenoviral vector-mediated rescue of the OMP-null phenotype in vivo. Nat Neurosci 3:1113-1120.

Jenkins PM, Hurd TW, Zhang L, McEwen DP, Brown RL, Margolis B, Verhey KJ, Martens JR (2006) Ciliary targeting of olfactory CNG channels requires the CNGB1b subunit and the kinesin-2 motor protein, KIF17. Curr Biol 16:1211-1216.

Jones BG, Thomas L, Molloy SS, Thulin CD, Fry MD, Walsh KA, Thomas G (1995) Intracellular trafficking of furin is modulated by the phosphorylation state of a casein kinase II site in its cytoplasmic tail. EMBO J 14:5869-5883.

Köttgen M, Benzing T, Simmen T, Tauber R, Buchholz B, Feliciangeli S, Huber TB, Schermer B, Kramer-Zucker A, Höpker K, Simmen KC, Tschucke CC, Sandford R, Kim E, Thomas G, Walz G (2005) Trafficking of TRPP2 by PACS proteins represents a novel mechanism of ion channel regulation. EMBO J 24:705-716.

Kulaga HM, Leitch CC, Eichers ER, Badano JL, Lesemann A, Hoskins BE, Lupski JR, Beales PL, Reed RR, Katsanis N (2004) Loss of BBS proteins causes anosmia in humans and defects in olfactory cilia structure and function in the mouse. Nature genetics 36:994-998.

Martin DM, Skidmore JM, Fox SE, Gage PJ, Camper SA (2002) Pitx2 distinguishes subtypes of terminally differentiated neurons in the developing mouse neuroepithelium. Dev Biol 252:84-99.

McEwen DP, Koenekoop RK, Khanna H, Jenkins PM, Lopez I, Swaroop A, Martens JR (2007) Hypomorphic CEP290/NPHP6 mutations result in anosmia caused by the selective loss of $\mathrm{G}$ proteins in cilia of olfactory sensory neurons. Proc Natl Acad Sci U S A 104:15917-15922.

Menco BP (1994) Ultrastructural aspects of olfactory transduction and perireceptor events. Semin Cell Biol 5:11-24.

Michalakis S, Reisert J, Geiger H, Wetzel C, Zong X, Bradley J, Spehr M, Hüttl S, Gerstner A, Pfeifer A, Hatt H, Yau KW, Biel M (2006) Loss of CNGB1 protein leads to olfactory dysfunction and subciliary cyclic nucleotidegated channel trapping. J Biol Chem 281:35156-35166.

Obara T, Mangos S, Liu Y, Zhao J, Wiessner S, Kramer-Zucker AG, Olale F, Schier AF, Drummond IA (2006) Polycystin-2 immunolocalization and function in zebrafish. J Am Soc Nephrol 17:2706-2718.

Otto EA, Loeys B, Khanna H, Hellemans J, Sudbrak R, Fan S, Muerb U, O’Toole JF, Helou J, Attanasio M, Utsch B, Sayer JA, Lillo C, Jimeno D, Coucke P, De Paepe A, Reinhardt R, Klages S, Tsuda M, Kawakami I, et al. (2005) Nephrocystin-5, a ciliary IQ domain protein, is mutated in Senior-Loken syndrome and interacts with RPGR and calmodulin. Nat Genet 37:282-288.

Pazour GJ, Rosenbaum JL (2002) Intraflagellar transport and ciliadependent diseases. Trends Cell Biol 12:551-555.

Piguet V, Wan L, Borel C, Mangasarian A, Demaurex N, Thomas G, Trono D (2000) HIV-1 Nef protein binds to the cellular protein PACS-1 to downregulate class I major histocompatibility complexes. Nat Cell Biol 2:163-167.

Ronnett GV, Moon C (2002) G proteins and olfactory signal transduction. Annu Rev Physiol 64:189-222. 
Rosenbaum JL, Witman GB (2002) Intraflagellar transport. Nat Rev Mol Cell Biol 3:813-825.

Schermer B, Höpker K, Omran H, Ghenoiu C, Fliegauf M, Fekete A, Horvath J, Köttgen M, Hackl M, Zschiedrich S, Huber TB, Kramer-Zucker A, Zentgraf H, Blaukat A, Walz G, Benzing T (2005) Phosphorylation by casein kinase 2 induces PACS-1 binding of nephrocystin and targeting to cilia. EMBO J 24:4415-4424.

Scholey JM (2003) Intraflagellar transport. Annu Rev Cell Dev Biol 19:423-443.

Scott GK, Gu F, Crump CM, Thomas L, Wan L, Xiang Y, Thomas G (2003)

The phosphorylation state of an autoregulatory domain controls PACS1-directed protein traffic. EMBO J 22:6234-6244.
Scott GK, Fei H, Thomas L, Medigeshi GR, Thomas G (2006a) A PACS-1, GGA3 and CK2 complex regulates CI-MPR trafficking. EMBO J 25:4423-4435.

Scott JW, Acevedo HP, Sherrill L (2006b) Effects of concentration and sniff flow rate on the rat electroolfactogram. Chem Senses 31:581-593.

Youker RT, Shinde U, Day R, Thomas G (2009) At the crossroads of homoeostasis and disease: roles of the PACS proteins in membrane traffic and apoptosis. Biochem J 421:1-15.

Youngentob SL, Pyrski MM, Margolis FL (2004) Adenoviral vectormediated rescue of the OMP-null behavioral phenotype: enhancement of odorant threshold sensitivity. Behav Neurosci 118:636-642. 